\title{
La contribution du spatial en France aux sciences de l'atmosphère et du climat
}

\author{
Cathy Clerbaux' ${ }^{1}$, Jean-Noël Thépaut ${ }^{2}$, Gilles Bergametti ${ }^{3}$, \\ Frédéric Parol ${ }^{4}$, Philippe Bougeault ${ }^{5}$, Cyril Crevoisier 6 \\ 1 Laboratoire atmosphères et observations spatiales (Latmos), \\ Institut Pierre-Simon Laplace, Université Pierre-et-Marie-Curie / CNRS, Paris \\ 2 Centre européen pour les prévisions météorologiques à moyen terme, \\ Reading, Royaume-Uni \\ 3 Laboratoire interuniversitaire des systèmes atmosphériques, \\ Institut Pierre-Simon Laplace, Université Paris-Est Créteil / \\ Université Paris-Diderot / CNRS, Créteil \\ 4 Laboratoire d'optique atmosphérique, Université de Lille 1 sciences \\ et technologies / CNRS, Villeneuve d'Ascq \\ 5 Centre national de recherches météorologiques, \\ Météo-France / CNRS, Toulouse \\ 6 Laboratoire de météorologie dynamique, Institut Pierre-Simon Laplace, \\ École polytechnique / CNRS, Palaiseau \\ cathy.clerbaux@latmos.ipsl.fr
}

\section{Résumé}

Depuis une trentaine d'années, les données satellitaires jouent un rôle déterminant pour l'observation de l'atmosphère terrestre et du climat, avec une contribution française et européenne importante. Cet article reprend les différentes étapes de la définition d'une mission spatiale d'observation de la Terre, donne un aperçu des variables qui peuvent être restituées à partir des données mesurées et présente les applications sociétales qui bénéficient de ces observations.

\section{Abstract}

The French spatial contribution to atmospheric and climate sciences

Over the last 30 years, satellite data have been playing a key role for the monitoring of the Earth's atmosphere and climate, with an important French and European contribution. This article summarizes the successive stages of a space mission for Earth's observation, describes the geophysical variables that can be derived from the measurements, and presents the societal benefits associated with these observations. es premiers satellites d'observation de la Terre et de son atmosphère sont apparus dans les années 1960, motivés par le besoin de disposer de prévisions météorologiques plus précises. Aujourd'hui, de très nombreux instruments embarqués sur satellites sont en orbite autour de la Terre (Capderou, 2012) et apportent des informations essentielles sur la plupart des paramètres géophysiques nécessaires à la compréhension du système Terreatmosphère. Ces paramètres renseignent sur la Terre solide (comme le champ de gravité et ses variations), la surface terrestre, la végétation, les océans, les glaces, les gaz et les aérosols qui composent l'atmosphère, les nuages et les précipitations, et les flux radiatifs. Le tableau 1 reprend les paramètres géophysiques qui peuvent être estimés à partir des observations satellitaires, en utilisant différentes techniques d'observation couvrant une large gamme de longueurs d'onde (figure 1). Aucun instrument ne permet de mesurer simultanément toutes les variables importantes pour la connaissance de l'atmosphère et du climat, et la définition d'une mission résulte bien souvent d'une série de compromis scientifiques et technologiques.

Tableau 1. Variables mesurées pour les différentes composantes du système Terre-atmosphère et les services opérationnels associés.

\begin{tabular}{lll} 
Variables pertinentes & Service opérationnel associé \\
$\begin{array}{l}\text { Surfaces } \\
\text { continentales }\end{array}$ & $\begin{array}{l}\text { Végétation, échanges d'énergie, } \\
\text { paramètres biophysiques, état des sols, } \\
\text { cultures, indice de surface foliaire, etc. }\end{array}$ & $\begin{array}{l}\text { Surveillance des surfaces végétales et agricoles, } \\
\text { et du cycle hydrologique continental }\end{array}$ \\
\hline Océan & $\begin{array}{l}\text { Niveau de la mer, couleur de l'eau, vagues, } \\
\text { salinité, glace de mer, température de surface, } \\
\text { vent, etc. }\end{array}$ & $\begin{array}{l}\text { Alertes tempêtes, prévision marine, } \\
\text { routage des bateaux, zones de pêche, etc. }\end{array}$ \\
\hline $\begin{array}{l}\text { Composition } \\
\text { atmosphérique }\end{array}$ & $\begin{array}{l}\text { Concentrations en gaz à effet de serre, } \\
\text { concentrations en gaz réactifs, aérosols : } \\
\text { épaisseur optique et taille, etc. }\end{array}$ & $\begin{array}{l}\text { Qualité de l'air, surveillance de la couche d'ozone, } \\
\text { surveillance des panaches volcaniques (aviation) }\end{array}$ \\
\hline $\begin{array}{l}\text { Météorologie } \\
\text { et climat }\end{array}$ & $\begin{array}{l}\text { Températures, vapeur d'eau, flux radiatifs } \\
\text { (solaire et IR), nuages, précipitations }\end{array}$ & $\begin{array}{l}\text { Prévision météorologique, projections climatiques, } \\
\text { études de processus }\end{array}$ \\
\hline
\end{tabular}


Ces observations satellitaires viennent compléter les mesures au sol (de routine ou ponctuelles lors de campagnes d'observations) et les observations réalisées à l'aide de dispositifs embarqués à bord de bateaux, ballons ou avions. Elles sont souvent moins précises qu'une mesure in situ, mais offrent l'avantage d'une couverture spatiale inégalée et, dans de nombreux cas, d'une répétitivité temporelle des observations très importante. Malgré la résolution spatiale souvent limitée par rapport à des mesures ponctuelles et locales, les observations spatiales sont néanmoins très utiles pour appréhender les transferts d'échelle et fournir les « conditions aux limites » qui permettent de comprendre une mesure dans un contexte plus global. Par exemple, lors
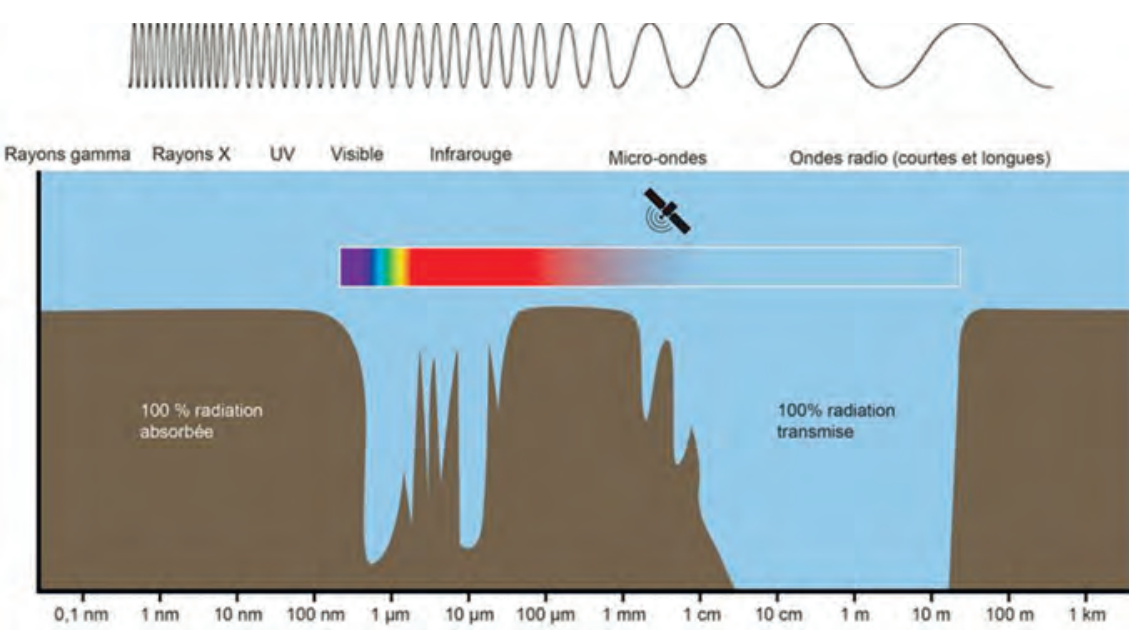

Figure 1. Gamme de longueurs d'onde couverte par le spectre électromagnétique, qui s'étend des rayons gamma aux ondes radios. Les ondes les plus et les moins énergétiques sont entièrement stoppées avant d'atteindre la surface terrestre. Le sondage de l'atmosphère par satellite repose sur les régions spectrales UV, visible, infrarouge, micro-ondes et radio. En particulier, les fenêtres atmosphériques optique et ondes radio sont exploitées pour sonder les couches les plus basses de l'atmosphère et la surface terrestre.

Tableau 2. Conclusions et recommandations du groupe " Océan, atmosphère, biosphère "lors du séminaire de prospective Cnes en 1989 (cap d'Agde). La mission Globsat n'a jamais existé, mais elle a donné naissance à différents concepts instrumentaux qui ont été lancés sur des satellites européens et américains.

Recommandations sur la programmation spatiale des années État des lieux actuel des instruments proposés 1995-2000. La première priorité : la mission Globsat

\begin{tabular}{|c|c|c|}
\hline $\begin{array}{l}\text { Spectromètre } \\
\text { infrarouge }\end{array}$ & $\begin{array}{l}\text { Profils de température } \\
\text { Profils d'humidité } \\
\text { Propriétés radiatives des surfaces } \\
\text { (nuages, sols...) } \\
\text { Contenus intégrés }\left(\mathrm{CH}_{4}, \mathrm{CO}, \mathrm{N}_{3} \mathrm{O}, \mathrm{O}_{3}\right)\end{array}$ & $\begin{array}{l}\text { Ce spectromètre infrarouge deviendra la mission } \\
\text { lasi, embarquée sur les satellites Metop-A } \\
\text { (2006) et B (2012) }\end{array}$ \\
\hline Gomos-T & $\begin{array}{l}\text { Profils de composants mineurs } \\
\text { Température et aérosols dans } \\
\text { la stratosphère (occultation stellaire) } \\
\text { Contenu intégré, profil d'ozone } \\
\text { (mode SBUV) }\end{array}$ & $\begin{array}{l}\text { Cet instrument sera lancé à bord du satellite } \\
\text { Envisat en } 2002\end{array}$ \\
\hline Sonia-C & $\begin{array}{l}\text { Imagerie visible/infrarouge des nuages } \\
\text { (résolution hectométrique, } \\
\text { échantillonnage kilométrique) }\end{array}$ & $\begin{array}{l}\text { L'instrument Meris sera lancé à bord du satellite } \\
\text { Envisat en } 2002 \text { (et l'équivalent américain Modis } \\
\text { à bord de Terra lancé par la Nasa en 1999) }\end{array}$ \\
\hline $\begin{array}{l}\text { Scarab } \\
\text { Radiomètre } \\
\text { couleur de l'eau }\end{array}$ & $\begin{array}{l}\text { Bilan radiatif } \\
\text { Chlorophylle, production primaire } \\
\text { eau côtière/écosystèmes marins } \\
\text { Échanges de } \mathrm{CO}_{2} \text { à l'interface air/mer } \\
\text { Cycle du carbone dans l'océan }\end{array}$ & $\begin{array}{l}\text { Scarab fera partie de la charge utile du satellite } \\
\text { Megha-Tropiques lancé en } 2011\end{array}$ \\
\hline \multicolumn{3}{|l|}{ Options } \\
\hline $\begin{array}{l}\text { Polder } \\
\text { Imageur polarimètre } \\
\text { vis-PIR }\end{array}$ & $\begin{array}{l}\text { Aérosols, biosphère continentale } \\
\text { et marine, effets directionnels } \\
\text { du bilan radiatif, nuages }\end{array}$ & $\begin{array}{l}\text { Ce concept donnera naissance à la filière Polder } \\
\text { sur les satellites japonais Adeos puis français } \\
\text { Parasol (A-Train) }\end{array}$ \\
\hline $\begin{array}{l}\text { Lired } \\
\text { Lidar rétrodiffusion }\end{array}$ & Altimétrie des nuages & $\begin{array}{l}\text { Le lidar Caliop volera à bord de la mission Calipso } \\
\text { en } 2006\end{array}$ \\
\hline
\end{tabular}

de la détection d'un épisode de pollution, l'observation satellitaire permet d'observer les sources impliquées et le déplacement des panaches de pollution.

\section{La genèse d'une mission satellitaire d'observation de la Terre}

La définition d'une mission satellitaire commence par l'identification des questions scientifiques ou des enjeux pour lesquels de nouvelles observations satellitaires peuvent apporter des informations pertinentes. Dans le domaine de l'atmosphère et du climat, ces questions concernent la compréhension des processus physiques, chimiques, biogéochimiques et dynamiques qui régissent les différents compartiments du système Terreatmosphère : surfaces continentales, végétation, océan, atmosphère y compris les nuages. Les satellites sont devenus incontournables pour apporter des informations permettant d'améliorer la prévision du temps, de suivre les principaux indicateurs du changement climatique (concentrations en gaz, stockage du carbone dans la végétation, niveau des mers, fonte des glaces...) et environnementaux (trou dans la couche d'ozone, pollution atmosphérique, érosion des sols, crues, couleur de la mer...).

La mise au point de chaque mission nécessite différentes étapes qui s'étalent sur plusieurs années, avec des points clés qui déterminent le passage à l'étape suivante ou l'arrêt de la mission. Le tableau 2 illustre l'histoire d'un concept de satellite français qui a émergé en 1989 et qui a conduit à une série d'instruments dont certains sont encore en vol.

\section{Définition des questions scientifiques à résoudre et des variables à observer}

Dans cette première étape, les scientifiques cherchent à identifier les questions pour lesquelles les observations spatiales apporteront une information pertinente et à définir les variables géophysiques à mesurer. Ces questions peuvent être d'ordre exploratoire (nouvelle technologie ou observation de nouvelles variables) ou 


\section{Empreinte au sol et trace d'une mesure au nadir}

La résolution spatiale est caractérisée par la taille de l'empreinte au sol (footprint, pixel size ou field of view) et la surface terrestre balayée à une fréquence donnée (trace, fauchée). Autrement dit, l'empreinte au sol est la surface terrestre couverte par une mesure individuelle effectuée par le sondeur. La trace au sol est une succession d'empreintes au sol et détermine les parties du globe couvertes par le satellite.

opérationnel (amélioration de l'existant, continuité, etc.). Cette étape va aussi conditionner la partie du globe terrestre qui sera observée (couverture globale ou régionale), la fréquence des observations souhaitée, la résolution spatiale (voir encadré 1) pour chaque observation et la précision requise pour que l'observation soit utile. Idéalement, on construit un simulateur d'observations (voir encadré 2), qui nécessite de modéliser la physique de la mesure et les paramètres restitués, afin de définir les spécifications instrumentales qui seront importantes pour assurer le succès de la mission par rapport aux objectifs initiaux.

\section{Mise au point d'un concept d'instrument, choix de l'orbite et de la géométrie d'observation}

Cette étape (appelée «phase zéro ») est menée par les scientifiques, en général en collaboration avec l'industrie et

\section{Expérience de systèmes d'observations simulés}

L'objectif d'une OSSE (Observing system simulation experiment) est de quantifier l'impact d'instruments futurs sur la qualité d'un système de prévision (par exemple météorologique, océanique, qualité de I'air, etc.). Une première simulation de modèle est tout d'abord effectuée afin de générer "la réalité ». Des observations sont ensuite simulées autour de cette réalité, à la fois pour les réseaux d'observations existants (en respectant leur densité et leur qualité) et pour le nouvel instrument à évaluer. Enfin, deux expériences d'assimilation, avec et sans ce nouvel instrument, permettent d'en mesurer l'impact en comparant les analyses et prévisions respectives à " la réalité » simulée. Ces expériences d'assimilation sont bien évidemment effectuées avec un modèle différent de celui utilisé pour générer «la réalité ». La calibration des OSSE (en particulier la vérification du réalisme des simulations des réseaux d'observations existants, des modèles d'assimilation) est fondamentale pour la crédibilité des résultats. l'agence spatiale française - le Centre national d'études spatiales (Cnes) - ou une autre agence spatiale. La première phase consiste à réfléchir à un concept d'instrument qui puisse fournir des observations à partir desquelles les variables géophysiques nécessaires seront dérivées. Il s'agit tout d'abord de choisir la gamme de longueurs d'onde qui convient, le type d'instrument (voir encadré 3), ses performances radiométriques, etc. Lors de cette étape, de nombreuses itérations sont nécessaires entre les partenaires, afin de ne pas définir un instrument trop lourd, trop compliqué ou trop cher, mais qui permette d'atteindre la précision souhaitée. Le choix de l'orbite est également important: les orbites basses autour de 600-800 km permettent d'assurer une couverture géographique globale (orbite polaire) ou centrée sur une bande de latitude donnée, tandis que l'orbite géostationnaire, vers $36000 \mathrm{~km}$, permet d'observer toujours la même partie du globe avec une fréquence d'observation plus élevée (Capderou,

\section{Instruments actif et passif}

Les sondeurs et imageurs dits passifs mesurent le rayonnement solaire réfléchi ou le rayonnement thermique émis par la surface terrestre et I'atmosphère dans des longueurs d'onde diverses (ultraviolet, visible, infrarouge et micro-onde). Les instruments dits actifs émettent un rayonnement et mesurent la quantité restituée après réflexion et diffusion dans l'atmosphère et /ou à la surface terrestre. La résolution verticale est supérieure dans le cas des instruments actifs et en ciel clair. Par exemple, dans le domaine visible, les lidars fournissent une information précise sur la base et le sommet des nuages semi-transparents, tandis que dans le domaine des hyperfréquences les radars fournissent des profils de pluie et de nuages. La trace au sol des instruments actifs est souvent limitée à quelques centaines de mètres, alors que celle des instruments passifs est de l'ordre de plusieurs centaines de kilomètres.
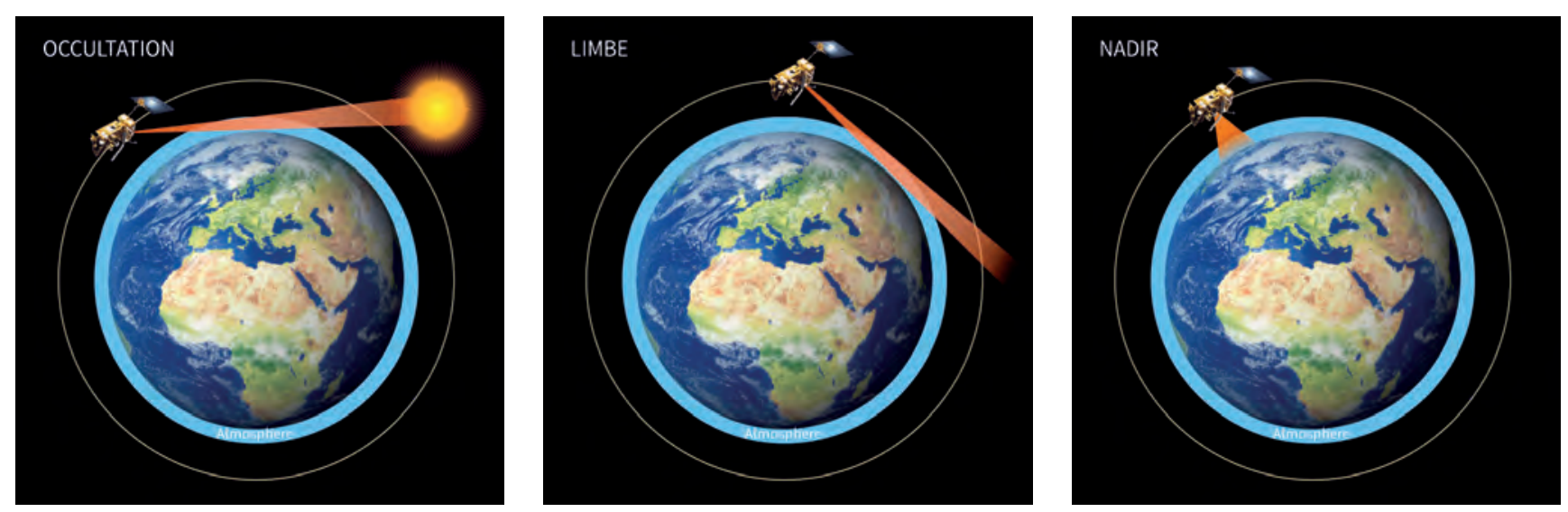

Figure 2. Trois géométries d'observation utilisées pour le sondage atmosphérique : (a) le sondage au limbe en occultation solaire qui vise le soleil (ou occultation lunaire ou stellaire, si on utilise la Lune ou une étoile comme source de rayonnement), (b) le sondage au limbe qui utilise comme source le rayonnement thermique et micro-ondes émis par l'atmosphère ou qui mesure le rayonnement UV-visible diffusé par la surface terrestre et l'atmosphère, et (c) le sondage au nadir qui pointe vers la surface terrestre et permet de sonder les plus basses couches de l'atmosphère. 
2012). Dans le cas du sondage de l'atmosphère, trois géométries d'observation sont possibles (figure 2) : les sondages au limbe (soit en occultation solaire, soit qui vise l'atmosphère) permettent de sonder la partie supérieure de l'atmosphère (typiquement la stratosphère et la mésosphère) avec une résolution verticale fine, et les sondages au nadir qui permettent de sonder la troposphère jusqu'à la surface terrestre, avec une résolution verticale modérée.

\section{Choix de la plateforme pour embarquer l'instrument et opportunité de lancement}

Une fois le concept d'instrument établi au cours de la phase A (démonstration de la faisabilité), en concertation avec l'industrie, il faut réfléchir à une opportunité de lancement pour cette mission, soit sur une plateforme dédiée, soit à bord d'une plateforme comportant déjà plusieurs autres instruments. Cette dernière solution est souvent privilégiée, car elle permet de diminuer les coûts de lancement et de bénéficier d'une série de paramètres auxiliaires qui peuvent être utilisés simultanément (par exemple pour les missions de sondage atmosphérique embarquées à bord de satellites météorologiques). Une coopération internationale est un autre moyen de faire diminuer les coûts, en combinant plusieurs instruments complémentaires sur une même plateforme ou sur une même orbite, comme dans le cas de l'A-train décrit brièvement à la fin de cet article. L'instrument peut être un démonstrateur (technologique ou pour prouver un concept) ou une filière pour bénéficier d'une série d'observations pérennes et cohérentes (programme Copernicus discuté ci-dessous). Des plateformes plus petites (type microsatellites) peuvent être également privilégiées dans le cas de missions dédiées à une thématique donnée pouvant tirer profit à des constellations de satellites. Une fois que la mission est formellement acceptée, les phases B et $\mathrm{C}$ (phases de conception) démarrent. On rappelle ici l'utilité de réaliser des prototypes d'instruments, qui peuvent voler sur des avions ou des ballons pour conforter la préparation d'un instrument spatial. Ces étapes permettent de finaliser les choix technologiques qui vont conduire à la construction de l'instrument. Elles précisent également la façon dont va se faire l'intégration de l'instrument sur la plateforme sélectionnée et dimensionnent les besoins du segment sol ${ }^{1}$ qui va réceptionner les données.

\section{Construction du modèle de vol et lancement de la mission}

Une fois la conception consolidée, le modèle de vol est construit, testé et intégré sur sa plate-forme satellite (phase D). Cette étape prend en général plusieurs années, car l'intégration de plusieurs instruments sur un satellite s'avère souvent complexe. Après le lancement de la mission, il peut s'écouler plusieurs mois avant que les données ne soient réellement disponibles : c'est la phase de recette en vol qui a pour objet de vérifier le bon fonctionnement de l'instrument, d'établir une caractérisation détaillée des performances, de valider les mesures en les comparant à d'autres instruments (au sol, à bord d'avions et de ballons) et de régler les paramètres de traitement des données. La période correspondant à l'exploitation des données est appelée phase E.

\section{Les observations par satellite}

La télédétection spatiale est utilisée pour fournir des mesures à partir des observations par satellite, obtenues loin de la grandeur géophysique à mesurer. L'observation satellite initiale doit subir un traitement informatique pour être transformée en mesure; la mise au point de ces algorithmes nécessite souvent un long travail, reposant sur des connaissances acquises et des simulations des observations. Différentes techniques de restitution coexistent, en général fondées sur des techniques de minimisation (interpolation optimale, Tikhonov, etc.) ou d'apprentissages supervisés (par exemple les réseaux de neurones).

Dans un second temps, les variables restituées sont validées à l'aide d'autres systèmes d'observation (in situ ou embarqués à bord de bateaux, d'avions, de ballons ou de satellites); cette étape est parfois complexe étant donné que les grandeurs mesurées sont rarement exactement comparables.

Pour différencier le niveau de traitement des données, on parle de données de niveau $0,1,2,3$ ou 4. Les données de niveau 0 sont les données brutes, non retravaillées, telles qu'enregistrées par l'instrument. Les données de niveau 1 sont les données corrigées et étalonnées, qui sont les mesures de base utilisées par les scientifiques pour dériver les grandeurs à restituer. Les données de niveau 2, 3 et 4 sont respectivement les mesures à la localisation de l'observation, les mesures moyennées sur une grille homogène et les mesures combinées à d'autres sources d'information compatibles (mesures ou modèles). Il est à noter que le passage direct du niveau 1 au niveau 4 est possible; il est d'ailleurs effectué de manière opérationnelle dans les centres de prévision numérique du temps, grâce aux méthodes d'assimilation de données telles que les méthodes variationnelles ou de filtre de Kalman d'ensemble. La figure 3 fournit un exemple de ces différents stades pour dériver des cartes du trou dans la couche d'ozone en Antarctique à partir des observations fournies par la mission Iasi à bord du satellite Metop.

\section{Des données spatiales aux services opérationnels}

Pour répondre au besoin d'observations spatiales pérennes et homogènes, en particulier pour les variables climatiques, des filières instrumentales stables sont apparues ces dernières années. Le concept de «variable climatique essentielle» (Essential Climate Variable ou ECV) a été introduit par le Global Climate Observing System (GCOS, 2015, 2016). La Climate Change Initiative (ou CCI) de l'Agence spatiale européenne (ESA) vise à établir de longues séries temporelles de référence pour une cinquantaine de ces variables, dont la plupart reposent sur des données satellitaires (Dowell et al., 2013).

Par ailleurs, l'Union européenne et l'ESA (voir encadré 4) ont décidé, au travers d'une initiative conjointe, de doter l'Europe d'une capacité opérationnelle, autonome et accessible à tous en observation de la Terre.

1. Le segment sol désigne les éléments de contrôle du satellite quand il est en vol ainsi que la récupération des données, leur traitement éventuel et leur distribution. 
Interférogramme lasi (niveau 0)

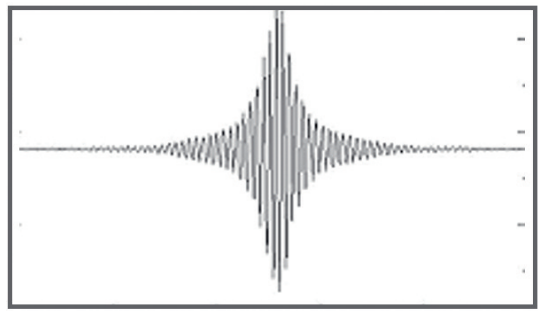

Carte d'ozone (niveau 2)

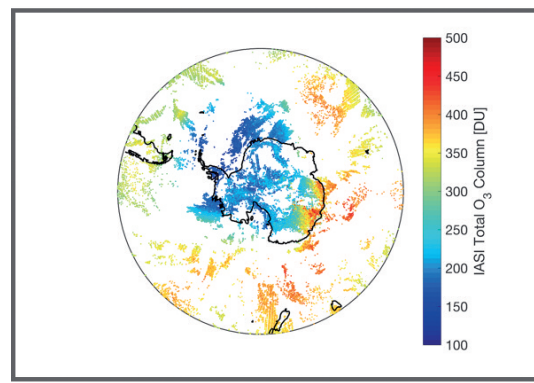

Spectre d'ozone

(niveau 1)

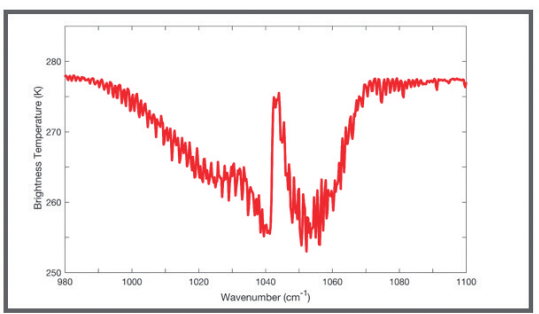

Carte d'ozone (niveau 3)

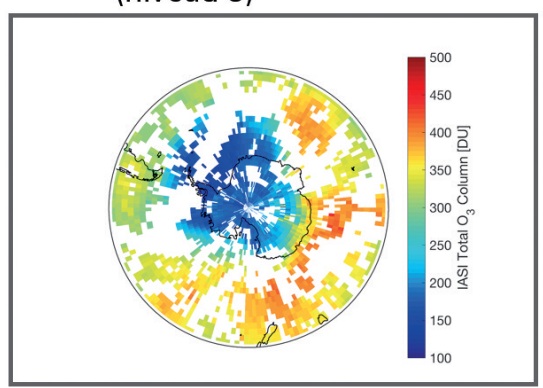

Profil d'ozone (niveau 2)

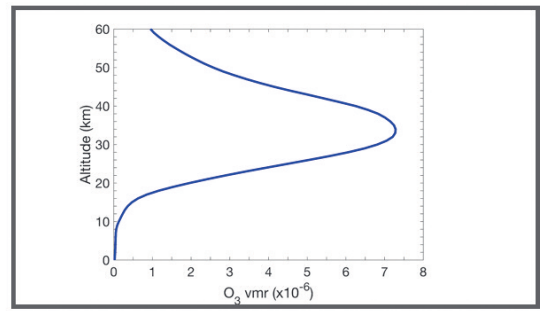

Carte d'ozone (niveau 4)

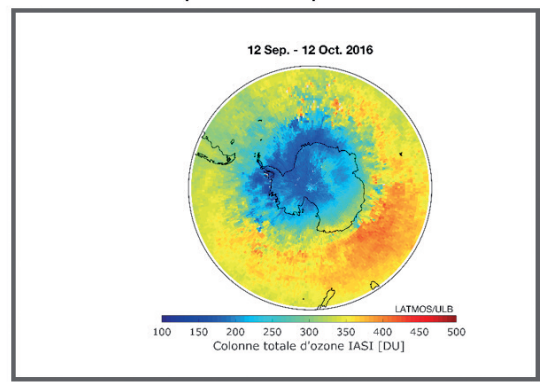

Figure 3. Les différentes étapes pour le traitement des données, du niveau 0 au niveau 4. L'exemple donné ici provient de la mission lasi où la donnée initiale est un interférogramme (niveau 0) converti en spectre atmosphérique avec une transformée de Fourier (niveau 1), puis en profil vertical d'ozone à la localisation de la mesure (niveau 2). La carte globale d'ozone peut être présentée à la résolution des observations (niveau 2) ou sous forme de moyenne (niveau 3), avant d'être assimilée dans un modèle de chimie-transport (niveau 4).

Ceci a été fait dans le cadre du programme européen de surveillance de la Terre Copernicus et a conduit à un fort rapprochement des communautés scientifiques « observation » et « modélisation » : les données d'observation (satellites, avions, ballons et in situ) sont aujourd'hui assimilées en temps réel dans des modèles pour offrir une série de services à valeur ajoutée à la société. La figure 4 représente de manière schématique le lien entre la composante satellitaire (baptisée Sentinelles, où chaque sentinelle regroupe une série de missions d'observation de la Terre), les variables mesurées pour caractériser le système Terre-atmosphère et les services opérationnels accessibles à tous.

\section{Le rôle de l'Europe et de la France}

La filière « observation de la Terre » par satellite a longtemps été dominée par les États-Unis, avec les programmes EOS et les trois satellites Terra, Aqua et Aura, lancés au début des années 2000, ainsi que via 1'A-train qui regroupe Aura et Aqua et une série d'autres missions, certaines françaises ou européennes ${ }^{2}$, qui se suivent rapidement sur une orbite similaire. Mais à la suite de choix politiques, en particulier celui de favoriser les missions d'exploration vers Mars, le nombre de missions de ce type a fortement diminué aux États-Unis, tandis que parallèlement l'Europe préparait le développement de ses satellites d'observation sur le long terme. L'Europe a fait le choix d'embarquer des sondeurs atmosphériques et des imageurs sur des plateformes météorologiques ; l'ESA et l'Eumetsat ${ }^{3}$ (voir encadré 4) ont travaillé de concert pour concevoir des systèmes intégrés, qui combinent des programmes en mode recherche (programme Earth Watch de l'ESA) et des programmes opérationnels (voir encadré 5) avec une continuité de service, permettant d'alimenter les services Copernicus.

Différents groupes de travail constitués de représentants du Cnes, de l'Insu et d'experts ont mené une réflexion sur l'avenir de la recherche spatiale en France dans le contexte actuel d'évolution du système de recherche (voir par exemple Puget et al., 2010). Le Cnes a eu et continue d'avoir un rôle important sur le développement de plusieurs missions qui ont volé ou volent encore, en particulier Jason (océanographie, voir Verron et Brasseur, 2017, ce numéro), Iasi (voir dans ce

2. Voir http://eospso.nasa.gov/

3. European organisation for the exploitation of meteorological satellites.

\section{ESA et Eumetsat}

L'Agence spatiale européenne (ESA) compte vingt-deux États membres qui mettent en commun leurs ressources pour développer les lanceurs, les véhicules spatiaux et les installations au sol dont l'Europe a besoin pour être autonome dans le domaine spatial. Les activités de l'agence couvrent l'astrophysique, l'exploration du Système solaire, l'étude du Soleil et la physique fondamentale, l'étude et I'observation de la Terre et de son atmosphère, le développement de lanceurs, les vols habités à travers sa participation à la Station spatiale internationale, la navigation par satellite avec le programme Galileo, les télécommunications spatiales et la recherche dans le domaine des technologies spatiales.

L'Organisation européenne pour I'exploitation des satellites météorologiques (Eumetsat) fédère 30 États membres européens (dont 26 des 28 de I'Union européenne) et a pour objectif principal la mise en place, la maintenance et l'exploitation des systèmes européens de satellites météorologiques. L'Eumetsat est responsable du lancement et des opérations des satellites, ainsi que d'acheminer les données aux utilisateurs finaux tout en contribuant à l'observation climatique et la détection des changements climatiques. Les services météorologiques des États membres financent les programmes $d^{\prime}$ Eumetsat et en sont les principaux utilisateurs. 

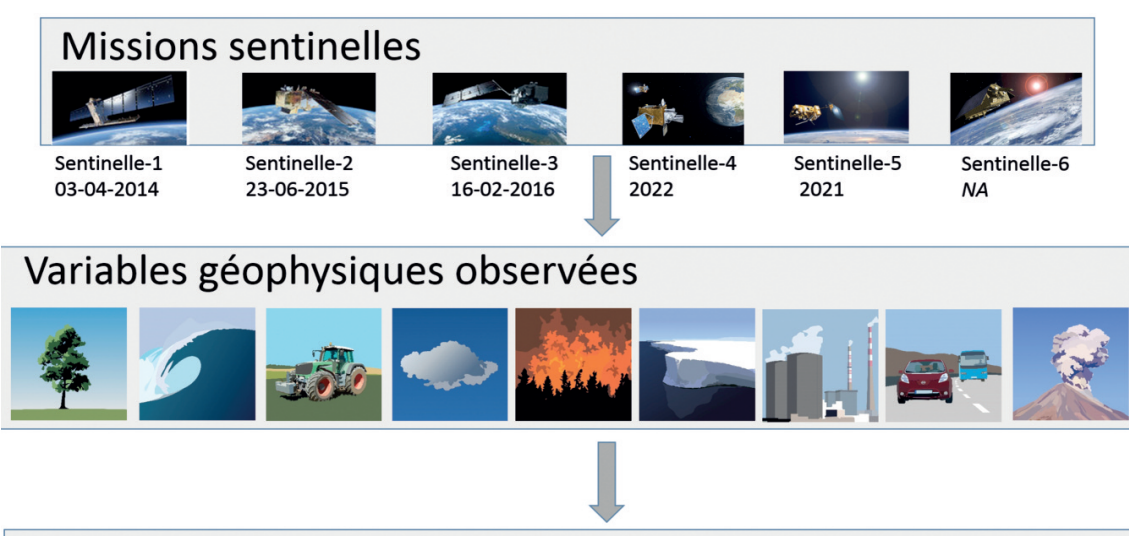

\section{Services opérationnels}

Terre Mer Atmosphère Urgences Sécurité Climat

Figure 4. Le programme Copernicus est une initiative européenne qui vise à rassembler les observations disponibles, couplées à des modèles, pour fournir une série de services opérationnels à la société. La composante spatiale repose sur six missions satellitaires appelées « sentinelles », dont les trois premières sont lancées et les trois suivantes seront lancées d'ici quelques années. Ces missions délivrent une série de variables qui permettent de caractériser l'état de la surface terrestre et de l'atmosphère pour alimenter des services opérationnels en aval : Terre (Land Monitoring Service), Mer (Marine Environment Monitoring Service), Atmosphère (Atmospheric Monitoring Service), Urgences (Emergency Management Service), Sécurité (Security Service) et Climat (Climate Change Service).

\section{Les satellites Metop et Meteosat}

La série de satellites Metop comporte trois satellites en orbite polaire, dont deux ont été lancés en 2006 et 2012 et dont le dernier sera lancé en 2018. II s'agit de plateformes météorologiques qui embarquent plusieurs instruments dont le sondeur UV-visible Gome-2 et le sondeur hyperspectral infrarouge Iasi. Le programme sera poursuivi après 2021 avec trois nouveaux satellites, Metop seconde génération, qui emporteront trois sondeurs atmosphériques : UVNS, Iasi-NG et $3 \mathrm{MI}$ (aérosols et nuages). Le programme Météosat quant à lui couvre l'orbite géostationnaire et, sur le même modèle, MTG (Meteosat troisième génération) embarquera des sondeurs dans les gamme de longueurs d'onde UV-visible et infrarouge, permettant pour la première fois le sondage atmosphérique à fréquence élevée au-dessus de l'Europe. Pour plus de détails sur ces missions, voir dans ce numéro les articles de Menut et al. (2017) et Stuhlmann et al. (2017).

\section{De Polder à $3 \mathrm{MI}$}

Développé par le Cnes, I'instrument spatial Polder est un polarimètre basé sur la combinaison d'une optique grand champ de vue (environ $\pm 55^{\circ}$ ), d'une roue porte-filtres spectraux (de 440 à $970 \mathrm{~nm}$ ) et porte-analyseurs, et d'un capteur matriciel CCD. II permet d'observer le rayonnement solaire réfléchi par une cible sous différents angles. Polder a été embarqué successivement sur deux plateformes spatiales japonaises, Adeos1 (1996) et Adeos2 (2003), et sur un microsatellite du Cnes, Parasol, qui a rejoint l'A-Train en décembre 2004 et a fourni neuf ans de mesures de polarisation et d'observations multi-angulaires sur les aérosols, les nuages et les surfaces continentales. Le concept de Polder a été récemment étendu à un domaine spectral plus large (de 410 à $2200 \mathrm{~nm}$ ) pour donner naissance à l'instrument $3 \mathrm{MI}$ qui sera embarqué sur les satellites opérationnels Metop-SC dès 2021 (voir figure 5).
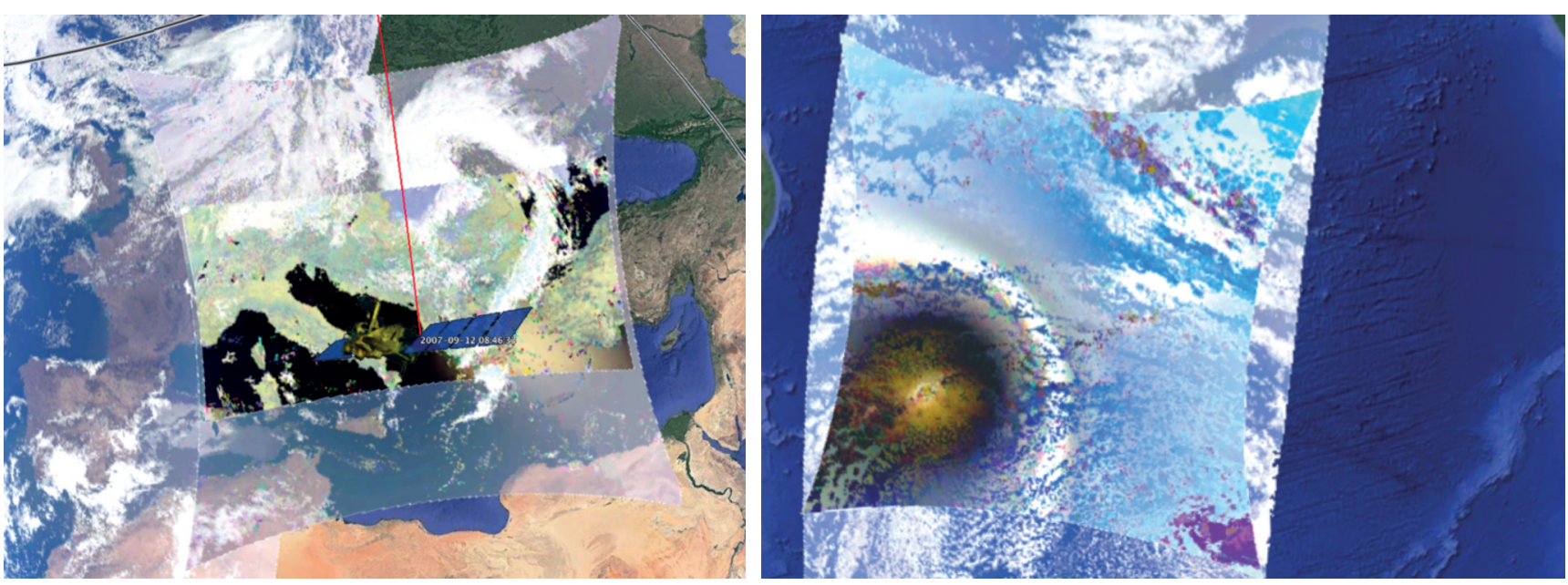

Figure 5. Images composites colorées illustrant les observations du futur 3MI sur Metop-SG obtenues à partir d'un simulateur de données synthétiques sur l'Europe (à gauche) et l'Atlantique Sud (à droite). L'image de gauche représente l'observation effectuée par l'instrument dans la partie visible du spectre solaire, ainsi que celle effectuée dans le proche infrarouge (petit rectangle au centre). La trace au sol du satellite est représentée en rouge. L'image de droite présente la lumière polarisée observée par l'instrument dans le domaine solaire. L'arc blanc et brillant témoigne de la présence de gouttelettes dans les nuages observés. 
d'informations sur ces deux dernières). La préparation des missions (avant le lancement) et l'exploitation des données (après le lancement) font l'objet de projets entre différents partenaires scientifiques avec des expertises complémentaires, qui travaillent sous l'égide du Cnes et du CNRS, notamment via le Tosca (groupe Terre-océan-surfaces continentales et atmosphère ; propositions de recherche scientifique soumises au Cnes et évaluées chaque année) et le PNTS (programme national de télédétection spatiale géré à l'Insu/CNRS).

\section{Conclusion}

La mise au point d'une mission satellitaire est une longue épopée qui requiert différentes étapes et des interactions fréquentes entre les scientifiques, les industriels et les agences spatiales. La qualité des données repose à la fois sur les spécifications instrumentales, mais aussi sur les algorithmes de traitement qui permettent d'extraire les grandeurs pertinentes des signaux enregistrés par le sondeur. Une fois que l'instrument est lancé, la phase de validation permet d'évaluer les données avant leur exploitation scientifique. La retransmission en temps réel des données, même pour les missions recherche, permet de garantir la détection la plus rapide possible des anomalies et d'accélérer le transfert vers l'opérationnel. Ces dernières années, l'initiative Copernicus a permis de fédérer différentes communautés autour d'un but commun pour offrir des services à valeurs ajoutées qui soient accessibles à tous les citoyens.

\section{Bibliographie}

Capderou M., 2012. Satellites : de Kepler au GPS, Springer.

Caudal G., Hauser D., Valentin R., Le Gac C., 2014. KuROS: A new airborne Ku-band Doppler radar for observation of surfaces. J. Atmos. Oceanic Technol., 31 , 2223-2245. doi: 10.1175/JTECH-D-14-00013.1

Chevallier F., Bousquet P., Bréon F.-M., Broquet G., 2017. Quantifier les puits et sources des gaz à effet de serre : une nouvelle ambition pour la télédétection spatiale. La Météorologie, 97, 46-51.

Clerbaux C., Boynard A., Clarisse L., George M., Hadji-Lazaro J., Herbin H., Hurtmans D., Pommier M., Razavi A., Turquety S., Wespes C., Coheur, P.-F., 2009. Monitoring of atmospheric composition using the thermal infrared IASI/MetOp sounder. Atmos. Chem. Phys., 9, 6041-6054.

Crevoisier C., Clerbaux C., Guidard V., Phulpin T., Armante R., Barret B., Camy-Peyret C., Chaboureau J.-P., Coheur P.-F., Crépeau L., Dufour G., Labonnote L., Lavanant L., Hadji-Lazaro J., Herbin H., Jacquinet-Husson N., Payan S., Péquignot E., Pierangelo C., Sellitto P., Stubenrauch C., 2014. Towards IASI-New Generation (IASI-NG): impact of improved spectral resolution and radiometric noise on the retrieval of thermodynamic, chemistry and climate variables. Atmos. Meas. Tech., 7, 4367-4385. doi: 10.5194/amt-7-4367-2014

Dowell M., Lecomte P., Husband R., Schulz J., Mohr T., Tahara Y., Eckman R., Lindstrom E., Wooldridge C., Hilding S., Bates J., Ryan B., Lafeuille J., Bojinski S., 2013. Strategy towards an architecture for climate monitoring from space. Available from: www.ceos.org/document-management/Working Groups/WGClimate/ WGClimate_Strategy-Towards-An-Architecture-For-Climate-Monitoring-From-Space_2013.pdf

Gaultier L., Ubelmann C., Fu L.-L., 2016. The challenge of using future SWOT data for oceanic field reconstruction. J. Atmos. Oceanic Technol., 33, 119-126. doi: 10.1175/JTECH-D-15-0160.1

GCOS, 2015. Status of the Global Observing System for Climate - Report 2015. Disponible à l'adresse : www.wmo.int/pages/prog/gcos/Publications/GCOS-195_en.pdf GCOS, 2016. GCOS implementation plan 2016. Disponible à l'adresse : http://unfccc.int/files/science/workstreams/systematic_observation/application/pdf/ gcos ip 10oct2016.pdf

Kraft S., Del Bello U., Bouvet M., Drusch M., Moreno J., 2012. FLEX: ESA's Earth Explorer 8 candidate mission. 2012 IEEE International Geoscience and Remote Sensing Symposium, 7125-7128. doi: 10.1109/IGARSS.2012.6352020

Mahfouf J.-F., Moisselin J.-M., Frédéric Autonès F., Vidot J., 2017. Apport de l'observation satellitaire pour la prévision du temps. La Météorologie, 97, 27-33.

Mandéa M., 2016. SWARM — Monitoring the Earth's magnetic field, CNES Rapport to COSPAR, 66-67.

Menut L., Attié J.-L., Beekmann M., Bessagnet B., Clerbaux C., Cuesta J., Dufour G., Forêt G., El Amraoui L., Mallet M., Meleux F., Ricaud P., Tanré D., Turquety S., 2017. Apport de l'observation satellitaire à l'analyse et à la prévision de la qualité de l'air. La Météorologie, 97, 34-45.

Puget J.-L. et al., 2010. Les sciences spatiales - Adapter la recherche française aux enjeux de l'Espace, Rapport de l'Académie des sciences sur la science et la technologie $n^{\circ}$ 30. Disponible à l'adresse : http://www.academie-sciences.fr/fr/Rapports-ouvrages-avis-et-recommandations-de-I-Academie/les-sciences-spatialesadapter-la-recherche-francaise-aux-enjeux-de-I-espace.html

Scipal K., Arcioni M., Chave J., Dall J., Fois F., LeToan T., Lin C.-C., Papathanassiou K., Quegans S., Rocca F., Saatchi S., Shugart H., Ulander L., Williams M., 2010. The BIOMASS mission - An ESA Earth Explorer candidate to measure the BIOMASS of the earth's forests. IEEE International Geoscience and Remote Sensing Symposium, 52-55. doi: 10.1109/IGARSS.2010.5648979

Stuhlmann R., Holmlund K., Schmetz J., Roquet H., Tjemkes S., Grandell J., Pohjola H., Lekouara M., Munro R., Fowler G., Schmid A., Aminou D., 2017. Observations depuis I'orbite géostationnaire avec Meteosat troisième génération (MTG) : continuité et innovation. La Météorologie, 97, 52-61.

Tanré D., Bréon F.M., Deuzé J.L., Dubovik O., Ducos F., François P., Goloub P., Herman M., Lifermann A., Waquet F., 2011. Remote sensing of aerosols by using polarized, directional and spectral measurements within the A-Train: the PARASOL mission. Atmos. Meas. Tech., 4, 1383-1395. doi: 10.5194/amt-4-1383-2011

Verron J., Brasseur P., 2017. Apports de l'observation satellitaire à l'océanographie. La Météorologie, 97, 62-69.

Winker D.M., Vaughan M.A., Omar A., Hu Y., Powell K.A., Liu Z., Hunt W.H., Young S.A., 2009. Overview of the CALIPSO mission and CALIOP data processing algorithms. J. Atmos. Oceanic Technol., 26, 2310-2323. doi: 10.1175/2009JTECHA1281.1

Zeng S., Parol F., Riédi J., Cornet C., Thieuleux F., 2011. Examination of POLDER/PARASOL and MODIS/Aqua cloud fractions and properties representativeness. J. Climate, 24, 4435-4450. doi: 10.1175/2011JCLI3857.1 